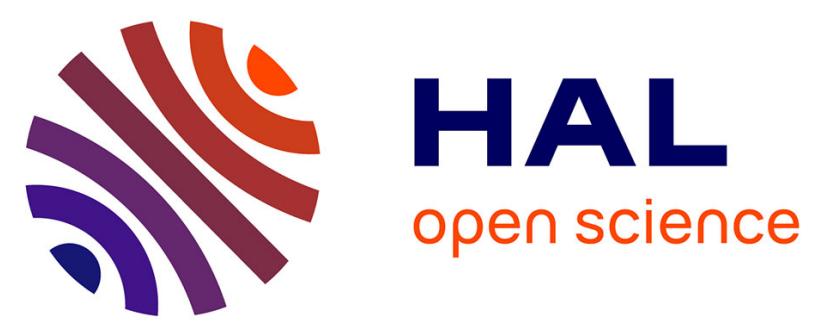

\title{
Influence of ligand substitution on molybdenum catalysts with tridentate Schiff base ligands for the organic solvent-free oxidation of limonene using aqueous TBHP as oxidant
}

\author{
Weili Wang, Dominique Agustin, Rinaldo Poli
}

\section{To cite this version:}

Weili Wang, Dominique Agustin, Rinaldo Poli. Influence of ligand substitution on molybdenum catalysts with tridentate Schiff base ligands for the organic solvent-free oxidation of limonene using aqueous TBHP as oxidant. Molecular Catalysis, 2017, 443, pp.52-59. 10.1016/j.mcat.2017.09.033 . hal-01940162

\author{
HAL Id: hal-01940162 \\ https://hal.science/hal-01940162
}

Submitted on 1 Mar 2021

HAL is a multi-disciplinary open access archive for the deposit and dissemination of scientific research documents, whether they are published or not. The documents may come from teaching and research institutions in France or abroad, or from public or private research centers.
L'archive ouverte pluridisciplinaire HAL, est destinée au dépôt et à la diffusion de documents scientifiques de niveau recherche, publiés ou non, émanant des établissements d'enseignement et de recherche français ou étrangers, des laboratoires publics ou privés. 
Influence of ligand substitution on molybdenum catalysts with tridentate Schiff base ligands for the organic solvent-free oxidation of limonene using aqueous TBHP as oxidant.

Weili Wang, ${ }^{a, b}$ Dominique Agustin*, ${ }^{a, b}$ Rinaldo Polia,c

${ }^{a}$ CNRS; LCC (Laboratoire de Chimie de Coordination); Université de Toulouse; UPS, INPT, 205, route de Narbonne, F-31077 Toulouse, France Fax: $+33-561553003$

${ }^{b}$ Université de Toulouse; Institut Universitaire de Technologie Paul Sabatier - Département de Chimie, Av. Georges Pompidou, BP 20258, F-81104 Castres Cedex, France E-mail: dominique.agustin@iut-t/se3.fr.

c Institut Universitaire de France, 1 rue Descartes, 75231 Paris, France

\section{Abstract}

The oxidation of limonene by aqueous TBHP has been analyzed in the presence of molybdenum complexes $\left[\mathrm{MoO}_{2} \mathrm{~L}\right]_{2}$ as catalysts with five different tridentate ligands $\mathrm{L}$ in the absence of organic solvents (greener reaction conditions). The ligands are based on a common salicylidene amino(thio)phenolate, $S A(T) P$, backbone with differences in the coordination sphere (ONO for $L=S A P$ vs. ONS for $L=S A T P$ ) or in the salicyl moiety functionalization by $\mathrm{OH}$ groups for the ONO ligands. The process gives a regioselective endocyclic epoxidation to a kinetically controlled 1:1 mixture of the cis-LimO and trans-LimO epoxides and/or the isomeric diols $a x$-LimD and eq-LimD by the subsequent ring opening in the presence of water, with a product distribution that depends on the ligand, reaction time and temperature. In combination with control experiments of the cis/trans-LimO ring opening, the investigations demonstrate the catalytic action of the metal complexes in both the epoxidation and the ring opening steps, with the cis-LimO stereospecifically producing the $a x$-LimD product and the less reactive trans-LimO leading to a 4:3 mixture of $a x$-LimD and $e q$-LimD. The ONS system $\left[\mathrm{MoO}_{2}(\mathrm{SATP})\right]_{2}$ exhibits the highest catalytic activity in both steps.

\section{Keywords}

Epoxidation / Molybdenum / Limonene / Solvent-free process / Ligand effect 


\section{Introduction}

The use of natural feedstock as chemical source is of constantly increasing interest. ${ }^{1}$ Terpenes, due to their diverse structures, represent an interesting available ore for several chemical transformations. ${ }^{2}$ As an example of terpene, limonene (Lim) is an inexpensive substrate obtained as waste from the orange juice industry. ${ }^{3,4}$ The limonene epoxides (LimOs) and diols (LimDs), obtained by oxidation of Lim (Scheme 1), are added-value renewable-based compounds, ${ }^{5,6}$ with applications in the synthesis of fragrances, flavors, food additives, or compounds with biological and therapeutic activity. ${ }^{7,8}$ Both LimO isomers, cis-LimO and trans-LimO, find applications in medicine, ${ }^{9}$ metal coatings, varnishes, printing inks, and in natural based polymers ${ }^{10}$ but exist in Nature in insufficient quantities to satisfy the needs. ${ }^{11}$ Both LimD isomers, eq-LimD and $a x$-LimD, generated from the LimOs by epoxide ring opening with water, ${ }^{12}$ are also precursors of interest for the pharmaceutical industry. ${ }^{13}$

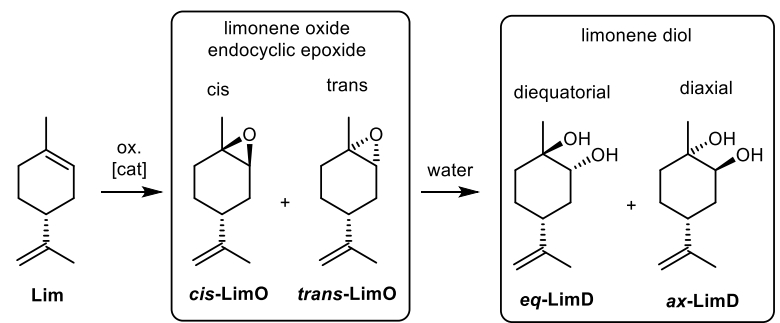

Scheme 1. Oxidation of limonene and potential LimO and LimD products.

The Lim epoxidation may occur at the internal and/or terminal alkene moiety and the presence of water facilitates the epoxide ring opening. In most cases, the main products are cis-LimO and trans-LimO deriving from the regioselective epoxidation of the endocyclic double bond, which is more reactive because of its higher electron density. The terms cis and trans, for this family of products, refer to the relative position of the $\mathrm{Me}$ and $\mathrm{iPr}$ substituents relative to the cyclohexane ring. Although four different stereoisomers may result in principle from the ring opening of cis-LimO and trans-LimO, only two of them, the diequatorial cis isomer (eq-LimD) and the diaxial trans isomer ( $a x$-LimD), have been obtained by this procedure as shown in Scheme 1 . Traces of other compounds such as carvone and carveol, resulting from allylic oxidation, may also be observed.

For the non-catalyzed processes leading to endocyclic epoxidation, organic peracids 
typically yield a 1:1 cis/trans-LimO mixture, ${ }^{14}$ whereas $\mathrm{N}$-sulfonyloxaziridines show stereoselectivities in favor of cis-LimO. ${ }^{15}$ In the extensive literature on metal catalyzed Lim epoxidation, the cis/trans stereoselectivity is rarely indicated. A variety of mononuclear molybdenum catalysts in combination with the TBHP oxidant were reported to yield equimolar amounts of the two isomers, ${ }^{16}$ while a greater stereoselectivity in favor of the trans isomer has been recently reported for a dinuclear Mo catalyst. ${ }^{16 \mathrm{~b}} \mathrm{~A}$ mononuclear iron catalyst yields predominantly the cis-LimO product. ${ }^{17}$

Concerning the selectivity of the ring opening step leading from LimO to LimD, it has been reported that both cis- and trans-LimO lead to the same $a x$-LimD product under acidic conditions $(\mathrm{pH} 3),{ }^{18}$ which was attributed to a selective axial nucleophilic attack that can be rationalized by the Fürst-Plattner rule. ${ }^{19}$ However, at an only slightly higher $\mathrm{pH}(4)$, only cisLimO is opened stereospecifically to yield $a x$-LimD while trans-LimO remains unreacted. ${ }^{18}$ Further studies have shown that the stereospecific opening of the cis-LimO isomer to axLimD also occurs in the presence of lanthanide ${ }^{20}$ or $\mathrm{Mo}^{21}$ catalysts. This ring opening selectivity has been exploited in some cases as a way to generate isomerically pure $a x$-LimD and/or to kinetically accumulate pure trans-LimO from the isomer mixture. The opposite preference for the trans-LimO ring opening has been accomplished by use of other conditions such as the use of nucleophilic amines, ${ }^{22}$ yielding again a trans-diaxial addition product, or water in the presence of mercuric reagents at $\mathrm{pH} \mathrm{7,} \mathrm{which} \mathrm{leads,} \mathrm{after} \mathrm{treatment}$ of an alkoxymercury intermediate with $\mathrm{NaBH}_{4}$, to the stereospecific generation of the eqLimD product. ${ }^{18,23}$ The LimO ring opening has also been explored with the use of enzymes. Using the Rhodotorula glutinis hydrolytic enzyme, optically pure $(1 S, 2 R, 4 S)$-cis-LimO was more rapidly and stereospecifically hydrolyzed to $(1 S, 2 R, 4 S)$-ax-LimD, whereas optically pure $(1 S, 2 R, 4 R)$-trans-LimO was opened more slowly to yield a mixture of $(1 R, 2 R, 4 R)$-eq-LimD $(65 \%)$ and $(1 S, 2 S, 4 R)$-ax-LimD (35\%). ${ }^{24}$ By direct enzymatic oxidation of Lim using a chloroperoxidase enzyme from Caldariomyces fumago, the LimD products were directly generated without detection of the LimO intermediates..$^{25}$ Only $a x$-LimD was selectively obtained when the procedure was carried out in the absence of $\mathrm{KCl}$ and proposed to result from the stereospecific opening of cis-LimO, whereas addition of $\mathrm{KCl}$ yields a mixture of $a x$ LimD and eq-LimD, proposed to derive from a mixture of cis-LimO and trans-LimO. These enzymatic studies are apparently the only ones highlighting the direct formation of eq-LimD 
by LimO ring opening.

It is also pertinent to highlight that the production of LimO and LimD has typically been accomplished using non-green conditions (oxidants such as peracids ${ }^{14}$ and/or organic solvents; ${ }^{26}$ or use of mercuric reagents for separation as highlighted above). In order to develop a greener access to these compounds, safer oxidants and non-hazardous conditions are required. ${ }^{27}$ Recently, we have reported the solvent-free epoxidation of cyclooctene and cyclohexene catalyzed by different molybdenum ${ }^{28}$ and vanadium ${ }^{29}$ complexes or by polyoxometalates. ${ }^{30}$ The concept was then further applied to terpenic compounds. ${ }^{31} \mathrm{~A}$ particular class of compounds that we have explored in some detail is the salicylidene amino(thio)phenolate family shown in Scheme 2. It was therefore of interest to apply these precatalysts to the Lim epoxidation under simpler and cleaner operating conditions, without organic solvents and with aqueous TBHP as oxidant. Preliminary results on the greener epoxidation of Lim using a few related Mo catalysts, yielding a mixture of cis-LimO, transLimo and $a x$-LimD, have been included in a recent publication. ${ }^{31 b}$ Here, we report a fuller investigation demonstrating how the ligand nature, particularly the presence of the sulfur atom or an $\mathrm{OH}$ group in the $\mathrm{R}_{1}$ position, affects the selectivity and the kinetics of the various transformations.

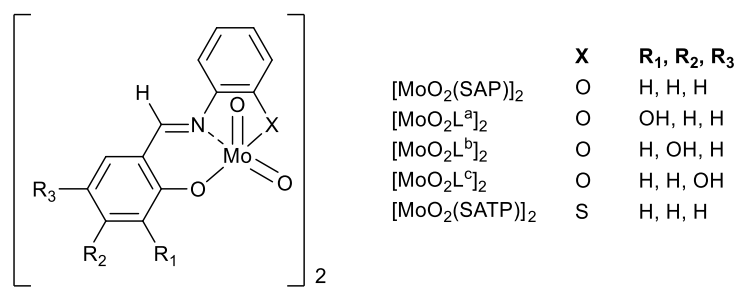

Scheme 2. Pre-catalysts used in this study

\section{Results and discussion}

\section{Catalyzed limonene oxidation}

The results of initial catalytic tests, run with all the pre-catalysts shown in Scheme 2, are collected in Table 1. In the absence of catalyst (run 1), aqueous TBHP leads only to a $7.6 \%$ conversion at $80^{\circ} \mathrm{C}$ after 4 hours, with detected traces of LimOs and LimDs. Using a $0.5 \%$ catalyst loading, Lim conversions of $79 \%$ (after 4 hours with $\left[\mathrm{MoO}_{2}(\mathrm{SAP})\right]_{2}$, run 2 ) and $93 \%$ (after 30 minutes with $\left[\mathrm{MoO}_{2}(\mathrm{SATP})\right]_{2}$, run 3 ) demonstrated the catalytic activity of both 
complexes as well as the greater activity of the sulfur-containing complex. The conversion monitoring for runs 1, 2 and 3 is shown in Figure 1 . The much higher activity for the catalyst with the ONS coordination sphere in Lim oxidation parallels that observed previously for the epoxidation of cyclooctene. ${ }^{28 b}$

Table 1. Oxidation of limonene in the presence of various Mo catalysts. ${ }^{a}$

\begin{tabular}{|c|c|c|c|c|c|c|c|c|c|}
\hline \multirow{3}{*}{ Run } & \multirow{3}{*}{ Catalyst } & \multirow{3}{*}{$\begin{array}{c}\text { Time } \\
\text { (h) }\end{array}$} & \multirow{3}{*}{$\begin{array}{c}\text { Lim } \\
\text { Conv } \\
(\%) \\
\end{array}$} & \multicolumn{4}{|c|}{ Selectivity $(\%)^{b}$} & \multirow{3}{*}{ TON } & \multirow{3}{*}{$\begin{array}{c}\text { Highest } \\
\text { TOF } \\
\left(h^{-1}\right)^{c}\end{array}$} \\
\hline & & & & \multicolumn{2}{|c|}{ LimO } & \multicolumn{2}{|c|}{$\operatorname{LimD}$} & & \\
\hline & & & & Cis & trans & eq & $a x$ & & \\
\hline 1 & - & 4 & 7.6 & 5.3 & 14.5 & 0.4 & 6.6 & - & - \\
\hline 2 & {$\left[\mathrm{MoO}_{2}(\mathrm{SAP})\right]_{2}$} & 4 & 79 & 14.6 & 25.1 & 2.3 & 33.5 & 158 & 130 \\
\hline 3 & {$\left[\mathrm{MoO}_{2}(\mathrm{SATP})\right]_{2}$} & 0.5 & 93 & 0 & 0 & 12.4 & 40.6 & 185 & 371 \\
\hline 4 & {$\left[\mathrm{MoO}_{2} \mathrm{~L}^{\mathrm{a}}\right]_{2}$} & 4 & 90 & 2.1 & 4.2 & 4.1 & 44.3 & 180 & 308 \\
\hline 5 & {$\left[\mathrm{MoO}_{2} \mathrm{~L}^{\mathrm{b}}\right]_{2}$} & 4 & 68 & 19.0 & 29.0 & 1.3 & 30.3 & 136 & 77 \\
\hline 6 & {$\left[\mathrm{MoO}_{2} \mathrm{~L}^{\mathrm{C}}\right]_{2}$} & 4 & 77 & 10.9 & 20.8 & 3.6 & 35.7 & 154 & 110 \\
\hline
\end{tabular}

${ }^{a}$ Reaction conditions: $[\mathrm{Mo}] /$ limonene/TBHP $=0.5 / 100 / 200, \mathrm{~T}=80^{\circ} \mathrm{C}$.

${ }^{b}$ Select $=\mathrm{n}_{\text {product }}$ formed $/ \mathrm{n}_{\text {Lim }}$ transformed obtained from GC analysis of the organic phase.

${ }^{c}$ TOF is calculated using the time interval with maximum slope in the conversion plot.

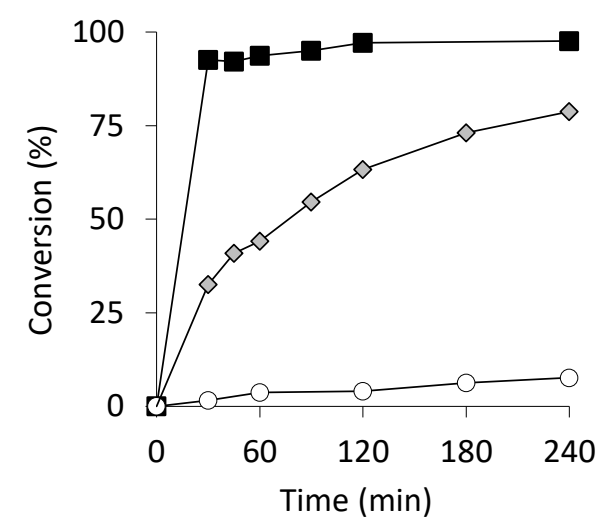

Figure 1. Kinetic profiles for limonene oxidation: without catalyst $(\mathrm{O})$, with $\left[\mathrm{MoO}_{2}(\mathrm{SAP})\right]_{2}(\diamond)$, with $\left[\mathrm{MoO}_{2}(\mathrm{SATP})\right]_{2}(\mathbf{\square})$. [Mo]/limonene/TBHP $=0.5 / 100 / 200, \mathrm{~T}=80^{\circ} \mathrm{C}$.

Monitoring the evolution of the various products in runs 2 and 3 revealed additional interesting features (see Figure 2). For the experiment with $\left[\mathrm{MoO}_{2}(\mathrm{SAP})\right]_{2}$ (Figure 2, left), the two LimO isomers are initially produced in a ca. $1: 1$ ratio ( $13.8 \%$ and $15.3 \%$ after $60 \mathrm{~min})$, but subsequently the amount of cis-LimO slowly decreased ( $2.6 \%$ after $4 \mathrm{~h}$ ) while that of transLimO continued to increase ( $19.8 \%$ after 4 h). These data indicate that the rate of cis-LimO ring opening exceeds that of its generation at longer reaction times, while trans-LimO opens the ring more slowly. At the same time, the $a x$-LimD amount increased from $4.7 \%$ to $26.5 \%$ 
while only traces of eq-LimD were detected initially and significant amounts are only formed after $3 h$.
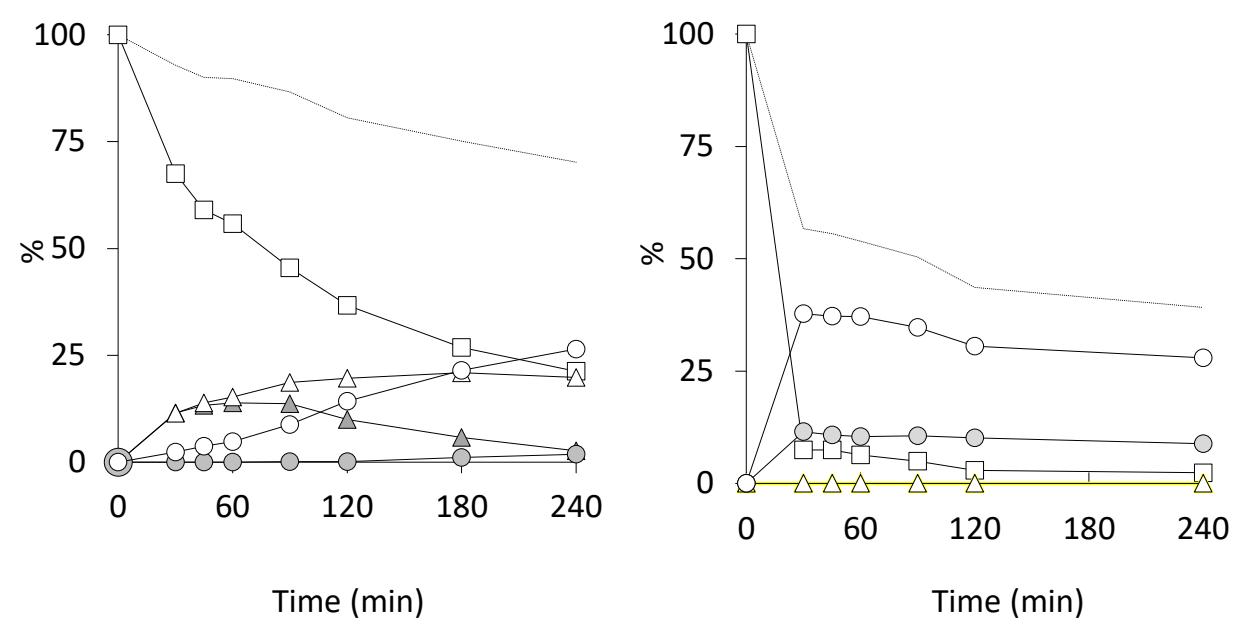

Figure 2. Kinetic profile for the limonene oxidation catalyzed by $\left[\mathrm{MoO}_{2}(\mathrm{SAP})\right]_{2}$ (left), $\left[\mathrm{MoO}_{2}(\mathrm{SATP})\right]_{2}$ (right): [Mo]/limonene/TBHP $=0.5 / 100 / 200, \mathrm{~T}=80^{\circ} \mathrm{C}$. Mass balance (...), limonene $(\square)$,cis-LimO $(\triangle)$, trans-LimO $(\triangle)$,eq-LimD (०),ax-LimD (O).

The initial observation of $a x$-LimD as the predominant diol in solution while only the cisLimO intermediate is being consumed at significant rate suggests that the faster ring opening of cis-LimO leads selectively to this product. This observation is consistent with previous literature reports. On the other hand, the slower generation of eq-LimD suggests that this isomer results from the slower ring opening of trans-LimO. We are unaware of other contributions where the direct generation of eq-LimD from metal-catalyzed Lim epoxidation has been highlighted (see also the Introduction). Whether the ring opening of trans-LimO is selective or leads to both diol products cannot be established from these data (see Scheme 3), but will be detailed later.

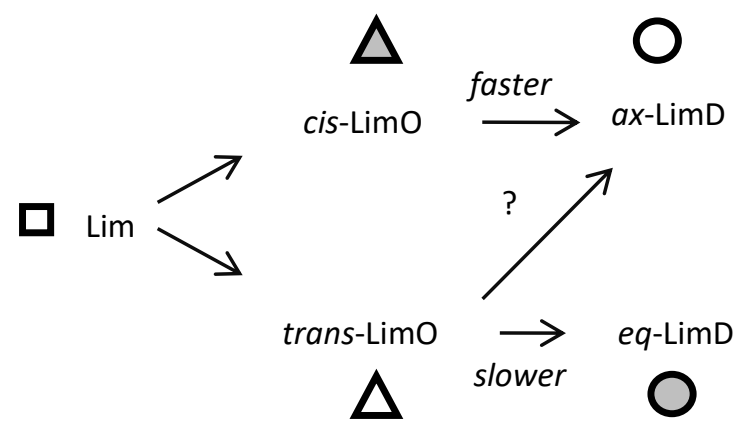


Scheme 3. Selectivities for the LimO ring opening

Concerning the experiment with $\left[\mathrm{MoO}_{2}(\mathrm{SATP})\right]_{2}$ (Figure 2, right), only traces of the intermediate epoxide compounds were detected, even at short reaction times, whereas the two isomeric diols were rapidly produced in large amounts. A greater amount of eq-LimD is generated relative to run 2 , although the $a x$-LimD isomer remains the prevalent product. These results suggest that not only the epoxidation step but also the ring opening step is metal catalyzed. These results are in line with a previous report, in which a $\left[\mathrm{MoO}_{2} \mathrm{Cl}_{2} \mathrm{~L}\right]$ complex ( $L=\beta$-ketophosphonate derived from camphor) was shown to catalyze the stereospecific ring opening of cis-LimO to yield $a x$-LimD, whereas trans-LimO remained essentially unreacted. ${ }^{21}$ The catalytic activity of $\left[\mathrm{MoO}_{2}(\mathrm{SAP})\right]_{2}$ and $\left[\mathrm{MoO}_{2}(\mathrm{SATP})\right]_{2}$ in the LimO ring opening has been further confirmed by separate investigations of the ring opening of cis- and trans-Limo by water (see further details in the next section).

The mass balance (cis-LimO + trans-LimO + eq-LimD $+a x$-LimD + residual $\operatorname{Lim}$ ) is low in both experiments: $80 \%$ after $2 \mathrm{~h}$ and $70 \%$ after $4 \mathrm{~h}$ for run 2; $56 \%$ after $0.5 \mathrm{~h}$ for run 3 . This loss of material can be attributed to the further oxidative transformations of the LimD isomers to non-volatile products. Traces of carvone and carveol, plus other unidentified products, were also visible in the gas-chromatogram for both runs and were not quantified but could be estimated as $<5 \%$, hence could not account for the lack of mass balance. The final reaction mixture consisted of a single phase in each case, presumably because the organic compounds and the water originating from the TBHP reagent solution, which generates two phases at the beginning of the reaction, are progressively compatibilized by the LimD and $t \mathrm{BuOH}$ products.

The kinetic profiles for the same oxidation processes in the presence of the precatalysts $\left[\mathrm{MoO}_{2} \mathrm{~L}^{\mathrm{a}-\mathrm{c}}\right]_{2}$ (runs 4-6) are shown in Figure 3 in comparison with those of runs 1 and 2 . The conversion after $4 \mathrm{~h}$ was very good for each catalyst (see Table 1 ), in the order $\left[\mathrm{MoO}_{2} \mathrm{~L}^{\mathrm{a}}\right]_{2}$ $(90 \%)>\left[\mathrm{MoO}_{2}(\mathrm{SAP})\right]_{2}(79 \%) \sim\left[\mathrm{MoO}_{2} \mathrm{~L}^{\mathrm{c}}\right]_{2}(77 \%)>\left[\mathrm{MoO}_{2} \mathrm{~L}^{\mathrm{b}}\right]_{2}(68 \%)$. The $\left[\mathrm{MoO}_{2} \mathrm{~L}^{\mathrm{a}}\right]_{2}$ complex $\left(\mathrm{R}_{1}\right.$ $=\mathrm{OH}$ ) exhibited a much higher activity than the other pre-catalysts. The initial cis/trans ratio for the LimO intermediates for the experiments with the $\left[\mathrm{MoO}_{2} \mathrm{~L}^{\mathrm{b}}\right]_{2}$ and $\left[\mathrm{MoO}_{2} \mathrm{~L}^{\mathrm{c}}\right]_{2}$ complexes was similar (ca. 1:1, see Figure 4) to that observed for the experiment with $\left[\mathrm{MoO}_{2}(\mathrm{SAP})\right]_{2}$ (Figure 2), whereas the most active $\left[\mathrm{MoO}_{2} \mathrm{~L}^{\mathrm{a}}\right]_{2}$ catalyst yields an initial cis/trans 
ratio of ca. 1:2 (Figure 4). Once produced, the LimO isomers are consumed to yield the two LimD products, mainly the axial isomer. The ring opening data are also in line with the behavior reported above for the parent $\left[\mathrm{MoO}_{2}(\mathrm{SAP})\right]_{2}$ pre-catalyst: rapid for cis-LimO with selective formation of $a x$-LimD and slower for trans-LimO (see further details later). In addition, the LimD selectivity correlates with the Lim conversion, with the relative amount of the minor eq-LimD product increasing with the rate of Lim conversion. These data further confirm that the epoxide ring opening process is, like the epoxidation, metal-catalyzed and that the better epoxidation catalyst is also a better ring opening catalyst. The same trends for the epoxide/diol selectivity vs. the rate of conversion, although quantitatively less pronounced, have also been previously observed for the oxidation of cyclohexene catalyzed by the same ONO complexes but no conclusion concerning the epoxide ring opening catalysis was made. ${ }^{28 \mathrm{~b}}$ The Green metrics for these catalyzed reactions have been analyzed in terms of the influence of catalyst and temperature on the LimO and LimD production (See $\mathrm{SI})$.

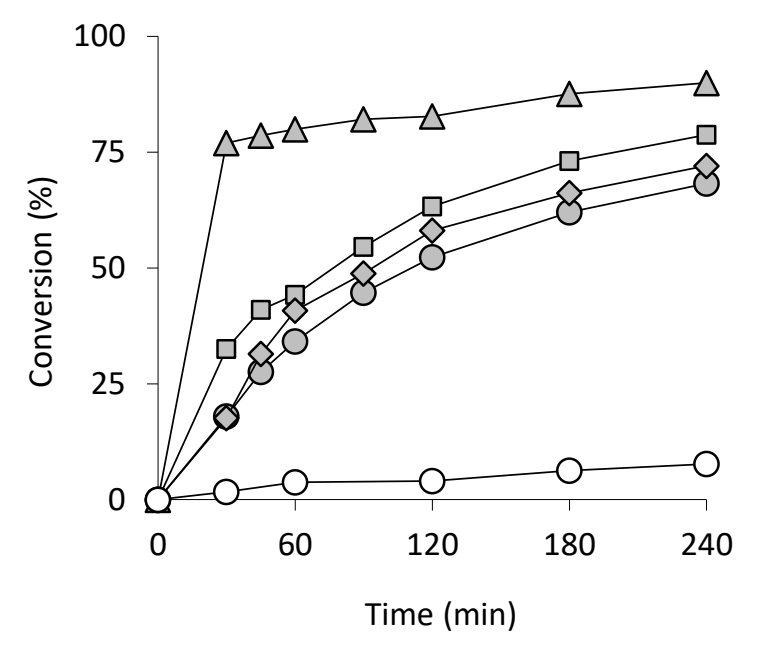

Figure 3. Kinetic profile for limonene oxidation: without catalyst $(-)$, with $\left[\mathrm{MoO}_{2}(\mathrm{SAP})\right]_{2}(\square)$, with $\left[\mathrm{MoO}_{2} \mathrm{~L}^{\mathrm{a}}\right]_{2}(\Delta)$, with $\left[\mathrm{MoO}_{2} \mathrm{~L}^{\mathrm{b}}\right]_{2}(\bullet)$, with $\left[\mathrm{MoO}_{2} \mathrm{~L}^{\mathrm{c}}\right]_{2}(\diamond)$. [Mo]/limonene/TBHP = $0.5 / 100 / 200$, reaction time $=4 \mathrm{~h}, \mathrm{~T}=80^{\circ} \mathrm{C}$. 

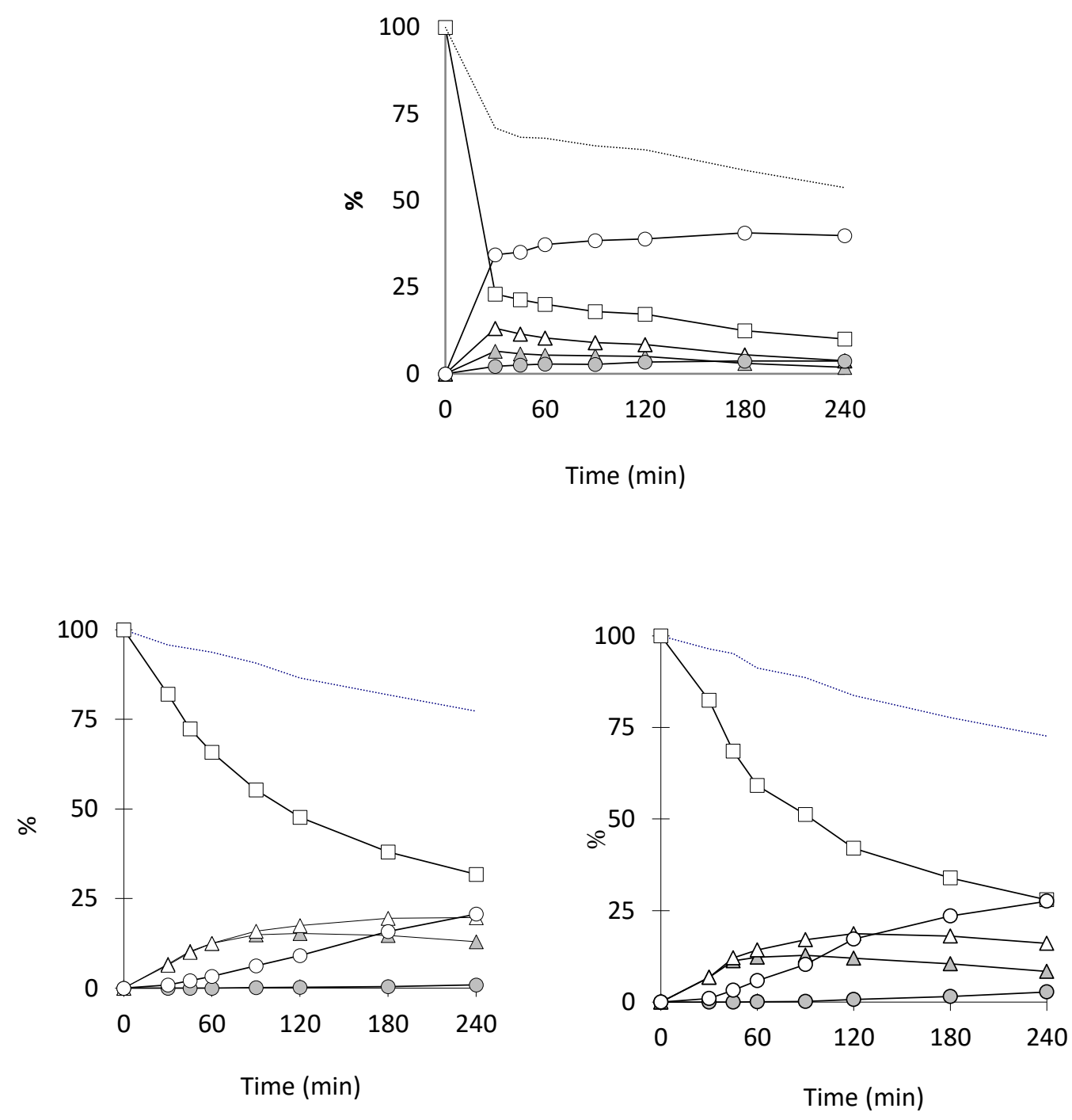

Figure 4. Kinetic profile for the limonene oxidation catalyzed by $\left[\mathrm{MoO}_{2} \mathrm{~L}^{\mathrm{a}}\right]_{2}$ (up), $\left[\mathrm{MoO}_{2} \mathrm{~L}^{\mathrm{b}}\right]_{2}$ (down left), $\left[\mathrm{MoO}_{2} \mathrm{~L}^{\mathrm{C}}\right]_{2}$ (down right), : [Mo]/limonene/TBHP $=0.5 / 100 / 200, \mathrm{~T}=80^{\circ} \mathrm{C}$. Mass balance $(. .$.$) , limonene (\square)$, cis-LimO $(\triangle)$, trans-LimO $(\triangle)$,eq-LimD $(\bullet)$, ax-LimD $(O)$.

In order to better understand the catalytic epoxidation and epoxide ring opening for this substrate, additional experiments were carried out for the more active $\left[\mathrm{MoO}_{2}(\mathrm{SATP})\right]_{2}$ systems at variable temperature and catalyst loading (Table 2 and Figures 5-7). A temperature increase without catalyst leads to greater Lim transformation although the conversion and selectivity remained poor (runs 9 and 12). With $0.5 \%$ molar ratio $\left[\mathrm{MoO}_{2}(\mathrm{SATP})\right]$ and 2 eq TBHP per substrate in the $30-80^{\circ} \mathrm{C}$ range, the Lim conversion after 4 $\mathrm{h}$ increased dramatically, from $36 \%$ at $30^{\circ} \mathrm{C}$ after $5 \mathrm{~h}$ to $93 \%$ at $80^{\circ} \mathrm{C}$ after $30 \mathrm{~min}$ (runs 8,11 
and 15; Figure 5). At both $50^{\circ} \mathrm{C}$ (runs $6-11$ ) and $80^{\circ} \mathrm{C}$ (runs 12-15) the conversion becomes faster as expected upon increasing the catalyst loading, see also Figure 6.

Table 2. Influence of temperature and catalyst loading for limonene epoxidation catalyzed by $\left[\mathrm{MoO}_{2}(\mathrm{SATP})\right]_{2} \cdot{ }^{a}$

\begin{tabular}{|c|c|c|c|c|c|c|c|c|c|c|}
\hline \multirow{3}{*}{ Run } & \multirow{3}{*}{$\begin{array}{c}\mathrm{T} \\
\left({ }^{\circ} \mathrm{C}\right)\end{array}$} & \multirow{3}{*}{$\begin{array}{l}\text { time } \\
\text { (h) }\end{array}$} & \multirow{3}{*}{$\begin{array}{c}X \\
(\%)\end{array}$} & \multirow{3}{*}{$\begin{array}{c}\text { Lim } \\
\text { Conv. } \\
(\%)\end{array}$} & \multicolumn{4}{|c|}{ Selectivity $(\%)^{b}$} & \multirow{3}{*}{ TON } & \multirow{3}{*}{$\begin{array}{c}\text { TOF } \\
\left(h^{-1}\right)^{c}\end{array}$} \\
\hline & & & & & \multicolumn{2}{|c|}{ LimO } & \multicolumn{2}{|c|}{ LimD } & & \\
\hline & & & & & Cis & trans & eq & $a x$ & & \\
\hline 7 & \multirow{2}{*}{30} & \multirow{2}{*}{5} & 0 & 3.2 & 9.4 & 9.4 & 1.6 & 5.6 & - & - \\
\hline 8 & & & 0.5 & 36 & 3.7 & 32.2 & 1.7 & 35.3 & 70 & 26 \\
\hline 9 & \multirow{3}{*}{50} & \multirow{3}{*}{5} & 0 & 5.3 & 10.4 & 0.6 & 0.6 & 5.8 & & \\
\hline 10 & & & 0.1 & 62.5 & 0.3 & 20.3 & 4.6 & 42.2 & 627 & 312 \\
\hline 11 & & & 0.5 & 83 & 0.1 & 10.4 & 5.8 & 50 & 166 & 161 \\
\hline 12 & \multirow{4}{*}{80} & 4 & 0 & 7.7 & 5.2 & 14.3 & 0.4 & 6.5 & & \\
\hline 13 & & 2 & 0.1 & 85 & 0 & 0 & 7.9 & 72.9 & 850 & 1042 \\
\hline 14 & & 0.5 & 0.25 & 87 & 0 & 0 & 11.4 & 46.3 & 348 & 1743 \\
\hline 15 & & 0.5 & 0.5 & 93 & 0 & 0 & 12.4 & 40.6 & 185 & 371 \\
\hline
\end{tabular}

${ }^{a}[$ Mo $] /$ limonene/TBHP $=x / 100 / 200 .{ }^{b}$

${ }^{b}$ Select $=n_{\text {product }}$ formed $/ n_{\text {Lim }}$ transformed obtained from GC analysis of the organic phase.

${ }^{c}$ TOF is calculated using the time interval with maximum slope in the conversion plot.

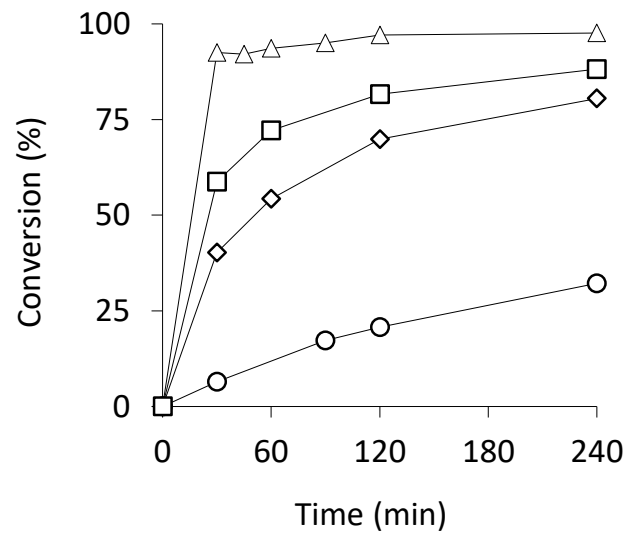

Figure 5. Influence of temperature on the $\left[\mathrm{MoO}_{2}(\mathrm{SATP})\right]_{2}$-catalyzed limonene oxidation: $30^{\circ} \mathrm{C}$ $(O), 50^{\circ} \mathrm{C}(\diamond), 60^{\circ} \mathrm{C}(\square), 80^{\circ} \mathrm{C}(\triangle)$. Conditions: [Mo]/limonene/TBHP = 0.5/100/200. 

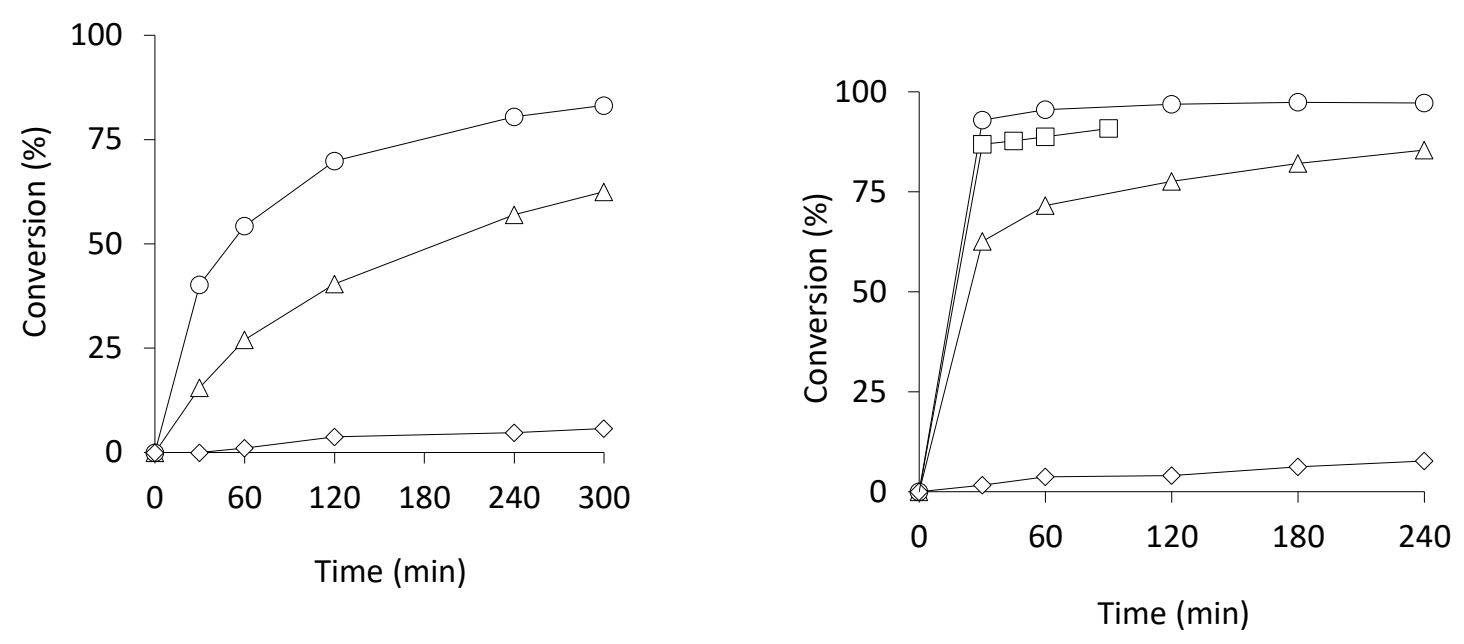

Figure 6. Influence of the catalyst loading on the $\left[\mathrm{MoO}_{2}(\mathrm{SATP})\right]_{2}$-catalyzed limonene oxidation at $50^{\circ} \mathrm{C}$ (left) and $80^{\circ} \mathrm{C}$ (right): [Mo]/limonene/TBHP $=x / 100 / 200, x=0(\diamond), 0.1(\triangle), 0.25(\square)$, $0.5(\mathrm{O})$.

The results of the individual intermediates and products monitoring at 30 and $50^{\circ} \mathrm{C}$ are shown in Figure 7. At the end of the catalytic runs, the LimO intermediates (mainly the trans isomer) could be detected only at temperatures up to $50^{\circ}$ when in the presence of catalyst. Interestingly, while the cis-LimO was the main epoxide product in the absence of catalyst at $50^{\circ} \mathrm{C}$, the selectivity was inverted in the presence of catalyst in favor of transLimO, probably because of the faster catalyzed ring opening of cis-LimO opening (mainly yielding $a x$-LimD as already discussed above). The highest eq-LimD yield was observed in the presence of catalyst at $80^{\circ} \mathrm{C}$ (run 15). 

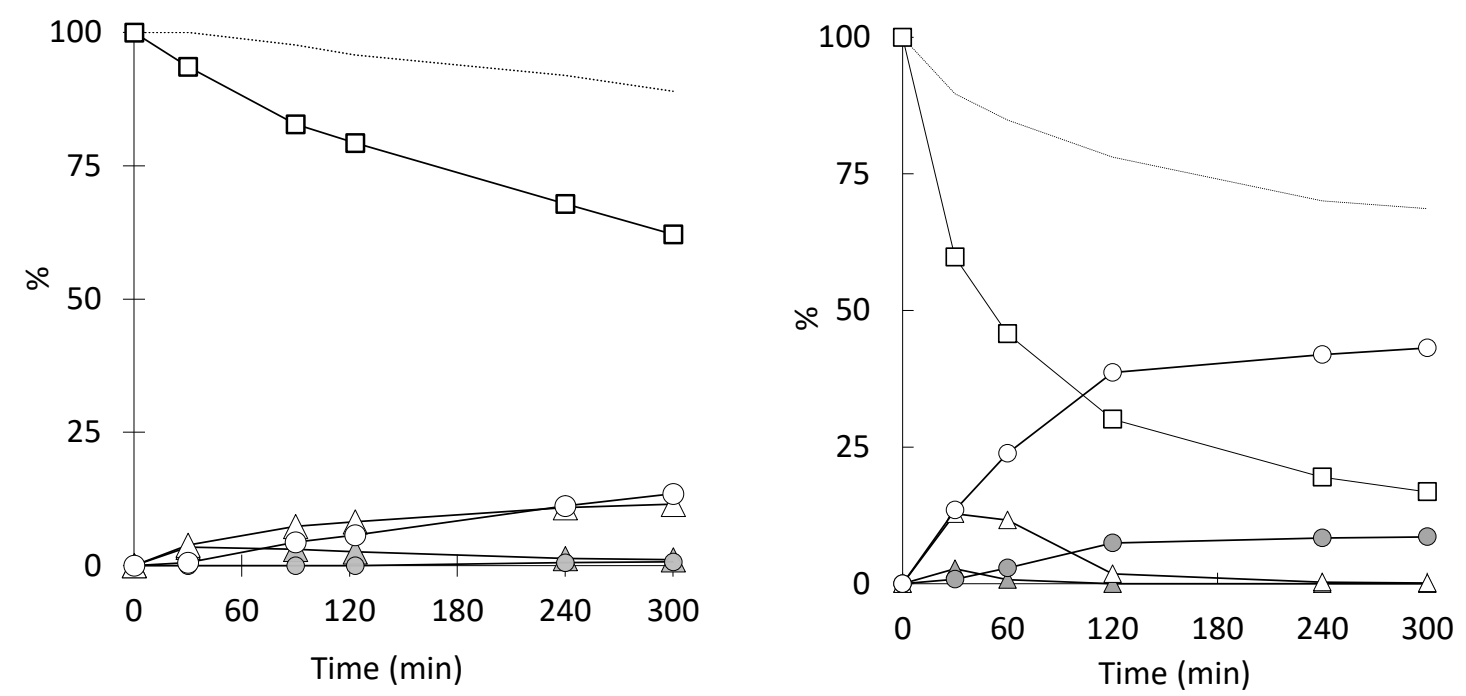

Figure 7. Kinetic profile for the limonene oxidation catalyzed by $\left[\mathrm{MoO}_{2}(\mathrm{SATP})\right]_{2}$ at $30^{\circ} \mathrm{C}$ (left) and $50^{\circ} \mathrm{C}$ (right): $[\mathrm{Mo}] /$ limonene/TBHP $=0.5 / 100 / 200$. Mass balance (...), limonene ( $\square$ ), cis$\operatorname{LimO}(\triangle)$, trans-LimO $(\triangle)$,eq-LimD $(\bullet)$,ax-LimD (O).

The effect of the TBHP/substrate ratio has also been investigated, both at $50^{\circ} \mathrm{C}$ and at $80^{\circ} \mathrm{C}$, with a $0.5 \%$ loading of the $\left[\mathrm{MoO}_{2}(\mathrm{SATP})\right]$ catalyst (Table 3). Lowering the TBHP amount leads, as expected, to a slower conversion (cf. run 16 with run 11 of Table 2 and run 17 with run 15 of Table 2). It is of interest to note that this also results in a slight decrease of the eqLimD selectivity and an increase of the $a x$-LimD selectivity.

Table 3. Influence of the TBHP amount on the $\left[\mathrm{MoO}_{2}(\mathrm{SATP})\right]_{2}$-catalyzed limonene epoxidation. ${ }^{a}$

\begin{tabular}{|c|c|c|c|c|c|c|c|c|c|c|}
\hline \multirow{3}{*}{ Run } & \multirow{3}{*}{$\mathrm{T}\left({ }^{\circ} \mathrm{C}\right)$} & \multirow{3}{*}{$\begin{array}{l}\text { Time } \\
\text { (h) }\end{array}$} & \multirow{3}{*}{$y$} & \multirow{3}{*}{$\begin{array}{c}\text { Conversion } \\
\text { (\%) }\end{array}$} & \multicolumn{4}{|c|}{ Selectivity $(\%)^{b}$} & \multirow{3}{*}{ TON } & \multirow{3}{*}{$\begin{array}{l}\text { TOF } \\
\left(h^{-1}\right)\end{array}$} \\
\hline & & & & & \multicolumn{2}{|c|}{ LimO } & \multicolumn{2}{|c|}{$\operatorname{LimD}$} & & \\
\hline & & & & & cis & trans & $e q$ & $a x$ & & \\
\hline 16 & 50 & 5 & 110 & 61 & 0 & 4.9 & 9.5 & 58.5 & 123 & 109 \\
\hline 17 & 80 & 0.5 & 150 & 89 & 0 & 0 & 12.2 & 47.4 & 178 & 357 \\
\hline
\end{tabular}

${ }^{a}$ Conditions: $[\mathrm{Mo}] /$ limonene $/ \mathrm{TBHP}=0.5 / 100 / \mathrm{y}$. Reaction time $=4 \mathrm{~h} . \mathrm{T}=50^{\circ} \mathrm{C}$.

${ }^{b}$ Select $=\mathrm{n}_{\text {product }}$ formed $/ \mathrm{n}_{\mathrm{Lim}}$ transformed obtained from GC analysis of the organic phase.

${ }^{c}$ TOF is calculated using the time interval with maximum slope in the conversion plot.

\section{Catalyzed limonene oxide ring opening}

As shown in the previous section, the Lim epoxidation produced LimOs and/or LimDs in a catalyst- and temperature-dependent process and there is clear evidence that the more 
active epoxidation catalysts (e.g. the SATP complex) also have higher activity in the ring opening process. It was therefore of interest to carry out more detailed investigations on the catalyzed epoxide ring opening process. For this purpose, both a commercial Limo isomer mixture (cis/trans $=47: 53$ ) and the pure individual isomers have been investigated (Table 4).

Table 4 - Ring opening of limonene oxides catalyzed by molybdenum complexes. ${ }^{a}$

\begin{tabular}{|c|c|c|c|c|c|c|c|c|c|}
\hline \multirow[t]{2}{*}{ Run } & \multirow[t]{2}{*}{ substrate } & \multirow[t]{2}{*}{$\begin{array}{l}\text { Time } \\
\text { (h) }\end{array}$} & \multirow[t]{2}{*}{ catalyst } & \multicolumn{2}{|c|}{ Conv (\%) } & \multicolumn{2}{|c|}{$\begin{array}{c}\text { LimD } \\
\text { selectivity } \\
\text { (\%) }\end{array}$} & \multirow[t]{2}{*}{ TON } & \multirow[t]{2}{*}{$\begin{array}{l}\text { TOF } \\
\left(\mathrm{h}^{-1}\right) \\
c\end{array}$} \\
\hline & & & & cis & trans & eq & $a x$ & & \\
\hline 18 & \multirow{3}{*}{$\begin{array}{l}\text { Limo } \\
\text { mixture }^{d}\end{array}$} & 4 & No catalyst & 60.7 & 12.0 & 11.7 & 58.7 & - & - \\
\hline 19 & & 4 & {$\left[\mathrm{MoO}_{2}(\mathrm{SAP})\right]_{2}$} & 93.3 & 26.2 & 2.6 & 58.5 & 119 & 45 \\
\hline 20 & & 0.5 & {$\left[\mathrm{MoO}_{2}(\mathrm{SATP})\right]_{2}$} & 100 & 100 & 12.1 & 45.0 & 200 & 400 \\
\hline 21 & \multirow{3}{*}{ cis-LimO } & 4 & No catalyst & 13.5 & & 0 & 6.4 & - & - \\
\hline 22 & & 4 & {$\left[\mathrm{MoO}_{2}(\mathrm{SAP})\right]_{2}$} & 26.9 & & 0 & 28.4 & 52 & 27 \\
\hline 23 & & 0.5 & {$\left[\mathrm{MoO}_{2}(\mathrm{SATP})\right]_{2}$} & 100 & & 0 & 25.3 & 200 & 400 \\
\hline 24 & trans-LimO & 1.5 & {$\left[\mathrm{MoO}_{2}(\mathrm{SATP})\right]_{2}$} & & 100 & 15.8 & 20.8 & 200 & 130 \\
\hline
\end{tabular}

${ }^{a}[\mathrm{Mo}] /$ limonene $/ \mathrm{TBHP}=0.5 / 100 / 200 ; \mathrm{T}=80^{\circ} \mathrm{C} .{ }^{b}$ Select $=\mathrm{n}_{\text {product }}$ formed $/ \mathrm{n}_{\text {Limo(cistrans) }}$ transformed obtained from GC analysis of the organic phase. ${ }^{c}$ TOF is calculated using the time interval with maximum slope in the conversion plot. ${ }^{d}$ Commercially available sample $($ cis/trans $=47 / 53)$.

The epoxidation of the trans/cis LimO mixture confirms that cis-LimO is opened faster than trans-LimO, whether in the presence or absence of catalyst. The rate of the transformation increases in the order: no catalyst (run 18) $<\left[\mathrm{MoO}_{2}(\mathrm{SAP})\right]_{2}$ (run 19) < $\left[\mathrm{MoO}_{2}(\mathrm{SATP})\right]_{2}$ (run 20). Both LimO isomers are opened faster by the more active $\left[\mathrm{MoO}_{2}(\mathrm{SATP})\right]_{2}$ catalyst (cf. runs 19 and 20$)$. The greater catalytic activity of the ONS ligand in ring opening is confirmed by the study of the isomerically pure cis-LimO (cf. run 22 and 23), which results in the stereospecific production of $a x$-LimD. Only the most active $\left[\mathrm{MoO}_{2}(\mathrm{SATP})\right]_{2}$ catalyst was tested in the opening of pure trans-LimO (run 24): in spite of the more sluggish ring opening of this isomer, quantitative conversion could be achieved after $1.5 \mathrm{~h}$. This run confirms that the eq-LimD isomer results from the opening of trans-LimO, but the product ratio is still in favor of the ax-LimD isomer. The mass balance suggests that either other reactions also occur, or that the LimD products are partially transferred in aqueous phase.

The separate investigations of pure cis-LimO and trans-LimO provide additional useful information on the stereochemistry of the ring opening process. None of the eq-LimD was generated by the ring opening of cis-LimO, either with or without catalyst (runs 21-23). Thus, 
both non-catalyzed and catalyzed cis-LimO ring openings lead stereospecifically to the $a x$ LimD isomer, in agreement with previous reports. ${ }^{18,21,24}$ However, the ring opening of pure trans-LimO (run 24) provides a mixture of $a x$-LimD and eq-LimD in a ca. 4:3 ratio. To the best of our knowledge, this is the first report of the metal-catalyzed opening of trans-LimO. Interestingly, the stereoselectivity of this Mo-catalyzed ring opening process is quite similar to that reported for the opening catalyzed by the hydrolytic enzyme Rhodotorula glutinis $(65: 35) \cdot{ }^{24}$

In terms of mechanistic interpretation, the generation of the eq-LimD product cannot result by an effect of the Mo complex identical to that proposed for $\mathrm{Hg}^{2+}$ in the selective generation of this diol from trans-LimO, for two reasons: $i$ ) the $\mathrm{Hg}^{2+}$ reagent accelerates the opening of trans-LimO relative to cis-LimO, whereas the Mo catalyst leads to faster opening of the cis isomer; ii) the $\mathrm{Hg}^{2+}$-promoted opening of the trans-LimO is stereospecific, leading solely to eq-LimD, whereas the Mo catalyst generates a mixture of both LimD isomers. Indeed, the action of $\mathrm{Hg}^{2+}$ was rationalized though a conformational change induced by the interaction between the soft $\mathrm{Hg}^{2+}$ ion and the isopropenyl substituent, which is not possible for the Lewis-acidic $\mathrm{Mo}^{\mathrm{VI}}$ center because this $d^{0}$ metal ion has no affinity toward $\mathrm{C}=\mathrm{C}$ unsaturations. We can rather invoke a mechanistic scheme with formation of $\mathrm{Mo}^{\mathrm{VI}}$-glycolate intermediates (see Scheme 4), based on that proposed by Cole-Hamilton et al. and supported by NMR investigations of the catalyst-substrate interactions. ${ }^{21}$ Activation of the epoxide by the Lewis acidity of the mononuclear 5-coordinate $\left[\mathrm{MoO}_{2} \mathrm{~L}\right]$ complex would result in equilibria with a molybdate-substituted carbocation and a Mo-glycolate intermediate (A and $\mathbf{B}$ from the cis isomer; $\mathbf{C}$ and $\mathbf{D}$ for the trans one). These intermediates are then attacked by water at the more substituted $\mathrm{C}$ atom with inversion of configuration. The smaller catalytic activity of the previously used camphor-based $\left[\mathrm{MoO}_{2} \mathrm{Cl}_{2} \mathrm{~L}\right]$ catalyst ${ }^{21}$ did not allow the stereoselectivity of the water addition to $\mathbf{D}$ to be established. Indeed, formation of intermediate $\mathbf{D}$ itself was not considered possible. We now find that the greater catalytic activity of $\left[\mathrm{MoO}_{2}(\mathrm{SATP})\right]$ (and to a certain extent also the other catalysts containing the SAP, $\mathrm{L}^{\mathrm{a}}, \mathrm{L}^{\mathrm{b}}$ and $\mathrm{L}^{\mathrm{c}}$ ligands) allows sufficiently rapid access to this intermediate. While the reactivity of the tertiary $\mathbf{C}$ atom in intermediate $\mathbf{B}$ dominates and leads to the selective generation of the trans-diaxial $a x$-LimD product, the lower reactivity of the same ring $C$ atom in intermediate D allows competitive attack at the secondary $\mathbf{C}$ atom with production of a mixture of the two LimD products. 


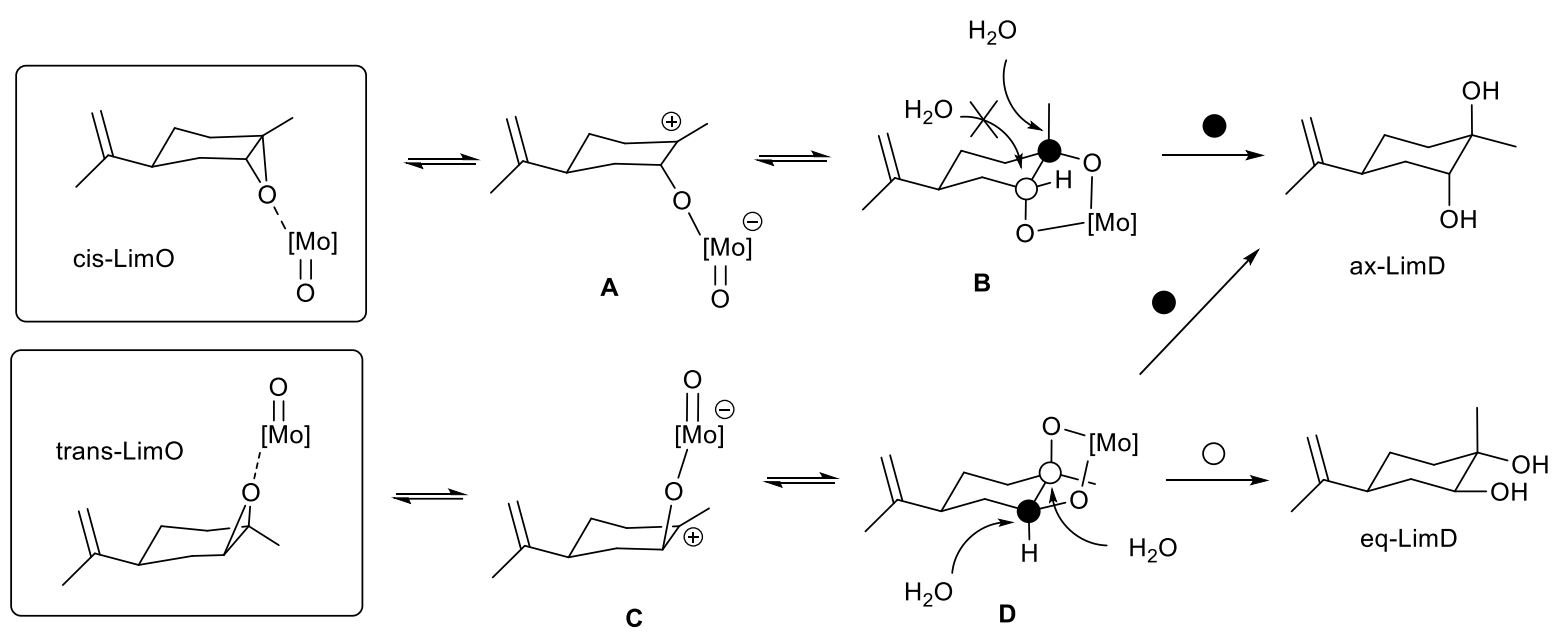

Scheme 4. Proposed mechanism for the Mo-catalyzed ring opening of the LimO isomers.

\section{Conclusions}

Lim epoxidation is catalyzed very efficiently by $\left[\mathrm{MoO}_{2} \mathrm{~L}\right]_{2}$ with salicylidene amino(thio)phenolate ligands, particularly $\left[\mathrm{MoO}_{2}(\mathrm{SATP})\right]_{2}$. These complexes promote not only the Lim regioselective endocylic epoxidation to yield both cis- and trans-LimO isomers in a kinetically controlled 1:1 ratio, but also the subsequent epoxide ring opening in the presence of water to obtain a mixture of $a x$ - and eq-LimD. The high activity of the $\left[\mathrm{MoO}_{2}(\mathrm{SATP})\right]_{2}$ precatalyst promotes the efficient ring opening of the less reactive transLimO isomer, which is shown to proceed unselectively to a mixture of $a x$ - and eq-LimD by analogy with a previous report of hydrolytic enzymatic ring opening, whereas the more reactive cis-LimO is stereospecifically opened to $a x$-LimD.

\section{Experimental part}

\section{Materials and methods}

All manipulations were carried out in air. Water was deionized twice before use. Organic solvents (ethanol, methanol, diethylether: synthesis grade, Aldrich) were employed as received without any purification. All molybdenum complexes were synthesized as previously described. ${ }^{28 a-c}$ Limonene (98\% Aldrich), the LimO mixture (Aldrich cis/trans 47/53, 98\%), ax-LimD (Aldrich, 98\%)) and TBHP (70\% in water, ACROS) were used as received. The pure cis-LimO, trans-LimO and eq-LimD were synthesized according to literature 
procedure. ${ }^{18,22,23}$ The catalytic reactions were monitored by gas chromatography on an Agilent 6890A chromatograph equipped with an FID detector, a HP5-MS capillary column $(0.30 \mathrm{~m} \times 0.25 \mathrm{~mm} \times 0.25 \mathrm{~m})$ and an autosampler, or on a Fisons GC 8000 chromatograph equipped with an FID detector and with a SPB-5 capillary column (30 $\mathrm{m} \times$ $0.32 \mathrm{~mm} \times 0.25 \mathrm{~m})$. Authentic samples of all reactants and products were used for calibration. The Lim conversion and the formation of LimOs and limDs were calculated from the calibration curves $\left(r^{2}=0.999\right)$ and an internal standard.

\section{Catalytic Procedure.}

In a typical experiment, limonene (1 equiv) and catalyst ( $x$ equiv, see tables) were mixed together in air in a round bottom flask. Acetophenone was added as internal standard. The flask was then immersed in a thermostated bath set at the desired temperature (see Tables) and magnetically stirred. After thermal equilibration, aqueous THBP (70\% in water, y equiv see tables) was added to the mixture, starting the reaction. Samples were periodically withdrawn and quenched by the addition of $\mathrm{MnO}_{2}$, followed by the addition of diethylether and removal of the manganese oxide and residual water by filtration through silica before GC analysis.

\section{Acknowledgements}

We acknowledge the Centre National de la Recherche Scientifique (CNRS) and the Institut Universitaire de France (IUF) for financial support, the Université Paul Sabatier and its Institut Universitaire Technologique for the facilities and the Chinese Scholarship Council for the fellowship of W.W. No.201208370239 


\section{References}

1 (a) G. A. Cordell, T. L. G. Lemos, F. J. Q. Monte, M. C. de Mattos, J. Nat. Prod. 70 (2007) 478-492. (b) A. Arbenz, L. Averous, Green Chem. 17 (2015) 2626-2646. (c) I. K M Yu , D. C W Tsang, Bioresource Technol. 238 (2017) 716-732. (d) A. K. Kumar, S. Sharma, Bioresources and bioprocessing 4 (2017) 7 (e) G. Chatel, K. De Oliveira Vigier, F. Jérôme, ChemSusChem 7 (2014) 2774-2787. (f) P. Spannring, P. C. A. Bruijnincx, B. M. Weckhuysen, R. J. M. Klein Gebbink, Catal. Sci. Technol. 4 (2014) 2182-2209 (g) C. O. Tuck, E. Perez, I. Horvath, R. Sheldon, M. Poliakoff, Science, 337 (2012) 695-699. (h) J. Kudakasseril Kurian, G. Raveendran Nair, A. Hussain, G. S. Vijaya Raghavan, Renew. Sust. Energ. Rev. 25 (2013) 205-219. (i) L. Filiciotto, A. M. Balu,, J. C. Van der Waal, R. Luque, Catal Today (2017) proofs online http://dx.doi.org/10.1016/j.cattod.2017.03.008 , (j) L. Wu, T. Moteki, A. A. Gokhale, D. W. Flaherty, F. D. Toste, Chemistry 1 (2016) 32-58. (k) F. D. Pileidis, M.-M. Titirici, ChemSusChem 9 (2016) 562-582. (I) V. Negro, G. Mancini, B. Ruggeri, D. Fino, Bioresource Technol. 214 (2016) 806-815. (m) E. V. Gusevskaya, ChemCatChem 6 (2014) 1506-1515. (n) T. I. J. Dugmore, J. H. Clark, J. Bustamante, J. A. Houghton, A. S. Matharu, Top Curr Chem 375 (2017) 46. (o) M. P. Adhikari, R. Adhikari, R. G. Shrestha, R. Rajendran, L. Adhikari, P. Bairi, R. R. Pradhananga, L. K. Shrestha, K. Ariga. Bull. Chem. Soc. Jpn. 88 (2015) 1108-1115. (p) V. S. Sikarwar, M. Zhao, P. Clough, J. Yao, X. Zhong, M. Z. Memon, N. Shah, E. J. Anthony, P. S. Fennell, Energy Environ. Sci. 9 (2016) 2939-2977. (q) A. Scoma, S. Rebecchi, L. Bertin, F. Fava, Crit. Rev. Biotechnol. 36 (2016) 175-189)

2 (a) W. Schwab, C. Fuchs, F.-C. Huang, Eur. J. Lipid Sci. Technol. 115 (2013) 3-8. (b) N. Ravasio, F. Zaccheria, M. Guidotti, R. Psaro, Top. Catal. 27 (2004) 157-168. J. L. F. Monteiro, C. O. Veloso, Top. Catal. 27 (2004) 169-180.

3 G. A. Burdock, Fenaroli's handbook of flavor ingredients, 3rd ed. CRC Press, Boca Raton. 1995 p.107.

4 (a) R. A. Sheldon, in: F. E. Harkes (Ed.) Catalysis of Organic Reaction, USA, 1998.

(b) R. A. Sheldon, in: G. Centi, F. Trifiro (Eds.) New Developments in Selective Oxidation, Elsevier, Amsterdam, 1990.

5 R. Ciriminna, M. Lomeli-Rodriguez, P. Demma Cara, J. A. Lopez-Sanchez, M. Pagliaro, Chem. Commun. 50 (2014) 15288-15296.

6 (a) K. A. D. Swift, Top. Catal. 27 (2004) 143-155.

7 (a) J. M. Derfer, M. M. Derfer, Encyclopedia of Chemical Technology Kirk-Othmer, vol.

22, third ed., Wiley, New York,1978, p. 709. (b) E. Herrero, S. Casuscelli, J. Fernandez, C.

Poncio, M. Rueda, O. Oyola, Molecules, 5 (2000) 336-337. (c) K. Bauer, D. Garbe, H.

Surburg, Common Fragrance and Flavor Materials, Preparation, Properties and Uses, Wiley VCH, New York, 1997.

8 (a) D. Comins, L. Guerra-Weltzien, J. M. Salvador, Synlett (1994) 972-974. (b) W.

Chrisman, J. N. Camara, K. Marcellini, B. Singaram, C. T. Goralski, D. L. Hasha, P. R.

Rudolf, L. W. Nicholson, K. K. Borodychuk, Tetrahedron Lett. 42 (2001) 5805-5807. (c) H. Lebel, E. N. Jacobsen, J. Org. Chem. 62 (1998) 9624-9625.

9 (a) S.K. Kulkarni, A. Dhir Prog. Neuropsychopharmacol. Biol. Psychiatry, 31 (2007) 12481254, (b) T. Umezu, Pharmacol. Biochem. Behav. 64 (1999) 35-40. (c) A. A. C. de 
Almeida, J. Pereira Costa, R. B. F. de Carvalho, D. Pergentino de Sousa, R. Mendes de Freitas, Brain Res. 1448 (2012) $56-62$

10 (a) C. M. Byrne, S.D. Allen, E. B. Lobkovsky, G. W. Coates, J. Am. Chem. Soc. 126 (2004) 11404-11405. (b) A. Corma, S. Iborra, A. Velty, Chem. Rev. 107 (2007) 2411-2502. (c) 0. Hauenstein, M. Reiter, S. Agarwal, B. Rieger, A. Greiner, Green Chem. 18 (2016) 760770. (d) E. Hosseini Nejad, A. Paoniasari, C. G. W. van Melis, C. E. Koning, R. Duchateau, Macromolecules 46 (2013) 631-637.

11 Jd. Moraes, A. A. Almeida, M. R. Brito, T. H. Marques, T. C. Lima, D. P. Sousa, E. Nakano, R. Z. Mendonça, R. M. Freitas, Planta Med. 79 (2013) 253-258

(a) C. K. Modi, J. A. Chudasam, H. D. Nakum, D. K. Parmar, A. L. Patel, J. Mol. Catal. A, Chem. 395 (2014) 151-161. (b) A. M. Santa A., J. C. Vergara G., L. A. Palacio, S., A. Echavarria, I. Catal. Today, 133-135 (2008) 80-86.

(a) W. F. Newhall, J. Org. Chem. 24 (1959) 1673-1676. (b) W. F. Newhall, J. Org. Chem. 29 (1964) 185-187. (c) W. Knoll, C. Tamm, Helv. Chim. Acta 58 (1975) 1162-1171.

(a) F. A. Davis, J. C. Towson, D. B. Vashi, R. Thimmareddy, J. P. McCauley, M. E. Harakal, D. J. Gosciniak, J. Org. Chem. 55 (1990) 1254-1261. (b) F. A. Davis, R. Thimmareddy, J. P. McCauley, R. M. Przeslawski, M. E. Harakal, P. J. Carroll, J. Org. Chem. 56 (1991) 809-815. (a) R. Clarke, M. Gahagan, R. K. Mackie, D. F. Foster, D. J. Cole-Hamilton, M. Nicol, A. W. Montford J. Chem. Soc., Dalton Trans. (1995) 1221-1226. (b) J. A. Brito, S. Ladeira, E. Teuma, B. Royo, M. Gomez, Appl. Catal. A 398 (2011) 88-95. D. Clemente-Tejeda, A. López-Moreno, F. A. Bermejo, Tetrahedron, 69 (2013) 29772986.

M. Blair, P. C. Andrews, B. H. Fraser, C. M. Forsyth, P. C. Junk, M. Massi, K. L. Tuck, Synthesis (2007) 1523-1527, and references cited therein.

(a) A. Fürst, P. A. Plattner, Helv. Chim. Acta 32 (1949) 275-283. (b) E. E. Royals, J. C. Leffingwell, J. Org. Chem. 31 (1966) 1937-1944.

P. C. Andrews, M. Blair, B. H. Fraser, P. C. Junk, M. Massi, K. L. Tuck, Tetrahedron: Asymmetry 17 (2006) 2833-2838.

L. Salles, A.F. Nixon, N. C. Russell, R. Clarke, P. Pogorzelec, D. J. Cole-Hamilton, Tetrahedron: Asymmetry 10 (1999) 1471-1476. Asymmetry 13 (2002) 2359-2363.

M. J. van der Werf, H. Jongejan, M. C. R. Franssen, Tetrahedron Lett. 42 (2001) 55215524.

C. Weijers, Tetrahedron : Asymmetry 8 (1997) 639-647.

S. Aguila, R. Vasquez-Duhalt, R. Tinoco, M. Rivera, G. Pecchi, J. B. Alderete, Green Chem 10 (2008) 647-653.

(a) A. A. Valente, I. S. Goncalves, A. D. Lopes, J.-E. Rodriguez-Borges, M. Pillinger, C. C. Romao, J. Rocha, X. Garcia-Mera, New J. Chem. 25 (2001) 959-963 (b) P. Oliveira, M. L. Rojas-Cervantes, A. M. Ramos, I. M. Fonseca, A. M. Botelho do Rego, J. Vital, Catal. Today 118 (2006) 307-314. (b) N. Mizuno, S. Hikichi, K. Yamaguchi, S. Uchida, Y. Nakagawa, K. Uehara, K. Kamata, Catal. Today 117 (2006) 32-36. (c) C. C. L. Pereira, S. S. Balula, F. A. Almeida Paz, A. A. Valente, M. Pillinger, J. Klinowski, I. S. Goncalves, Inorg. Chem. 46 (2007) 8508-8510. (d) T. R. Amarante, P. Neves, C. Tomé, M. Abrantes, A. A. Valente, F. A. Almeida Paz, M. Pillinger, I. S. Gonçalves, Inorg. Chem. 51 (2012) 
3666-3676. (d) T. R. Amarante, P. Neves, A. A. Valente, F. A. Almeida Paz, A. N. Fitch, M. Pillinger, I. S. Gonçalves, Inorg. Chem. 52 (2013) 4618-4628. (e) Z. Strassberger, E. V. Ramos-Fernandez, A. Boonstra, R. Jorna, S. Tanase, G. Rothenberg, Dalton Trans. 42 (2013) 5546-5553. (d) A. Gallo, C. Tiozzo, R. Psaro, F. Carniato, M. Guidotti, J. Catal. 298 (2013) 77-83. (e) J. Mlodzik, A. Wroblewska, E. Makuch, R. J. Wrobel, B. Michalkiewicz, Catal. Today, 268 (2016) 111-120. (f) A. Wroblewska, E. Makuch, P. Miadlicki, Catal. Today, 268 (2016) 121-129.

27 R. A. Sheldon, Green Chem. 7 (2005) 267-278.

28 (a) J. Morlot, N. Uyttebroeck, D. Agustin, R. Poli, ChemCatChem. 5 (2013) 601-611. (b) W. Wang, T. Vanderbeeken, D. Agustin, R. Poli, Catal. Commun. 63 (2015) 26-30. (c) W. Wang, J.-C. Daran, R. Poli, D. Agustin, J. Mol. Catal. A, 416 (2016) 117-126. (a) J. Pisk, D. Agustin, J. C. Daran, V. Vrdoljak, R. Poli, Adv. Synth. Catal. 353 (2011) 2910-2914. (b) J. Pisk, B. Prugovečki, D. Matković-Čalogović, R. Poli, D. Agustin,V. Vrdoljak, Polyhedron, 33 (2012) 441-449. (c) J. Pisk, B. Prugovečki, D. Matković-Čalogović, T. Jednačak, P. Novak, D. Agustin, V.Vrdoljak, RSC Adv. 4 (2014) 39000-39010. (d) V. Vrdoljak, J. Pisk, D. Agustin, P. Novak, J. Parlov Vuković, D. Matković-Čalogović. New J. Chem. 38 (2014) 6176-6185.

(b) J. Pisk, J.-C. Daran, R. Poli, D. Agustin, J. Mol. Catal. A: Chem. 403 (2015), 52-63. (2013) 3466-3475.

31 (a) M. Loubidi, D. Agustin, A. Benharref, R. Poli, C. R. Chim. 17 (2014) 549-556. (b) M. Cindric, G. Pavlovic, R. Katava, D. Agustin, New J. Chem. 41 (2017) 594-602. 